\title{
Erratum to: Evolution of Lipid Profiles after Bariatric Surgery
}

\author{
Ignacio Garcia-Marirrodriga • Cesar Amaya-Romero • \\ Gabriel Patiño Ruiz-Diaz • Sandra Férnandez • \\ Carlos Ballesta-López • Jose Ma. Pou • June H. Romeo • \\ Gemma Vilahur • Lina Badimon • Juan Ybarra
}

Published online: 21 December 2011

(C) Springer Science+Business Media, LLC 2011

\section{Erratum to: OBES SURG DOI 10.1007/s11695-011-0534-7}

In the original publication, the surname of the eighth author is incorrect. "Gemma Vilhur" should read "Gemma Vilahur."

In addition, the affiliations of the eighth and ninth authors was incomplete. The correct affiliations are:

Gemma Vilahur, $\mathrm{PhD}$

Cardiovascular Research Center (CSIC-ICCC), Institut Català de Ciències Cardiovasculars (ICCC), CIBEROBNPathophysiology of Obesity and Nutrition, Hospital Sta. Creu i St. Pau, Av. Sant Antoni M. Claret 167, 08025 Barcelona, Spain

The online version of the original article can be found at http://dx.doi. org/10.1007/s11695-011-0534-7.

\footnotetext{
I. Garcia-Marirrodriga • C. Amaya-Romero • G. P. Ruiz-Diaz •

C. Ballesta-López

Centro Laparoscópico Dr. Ballesta (CLB), Centro Médico Teknon,

08022 Barcelona, Spain
}

\section{S. Férnandez}

Resistencia a la Insulina SL,

08035 Barcelona, Spain

\section{J. Ybarra $(\bowtie)$}

Servicio de Endocrinología y Nutrición, Clinica CIMA,

08034 Barcelona, Spain

e-mail: juanybarra@hotmail.com

\section{J. H. Romeo}

Baldwin-Wallace College,

Berea, OH, USA

J. Ybarra

Endocrinology Department, Hospital de la Santa Creu i de Sant

Pau,

Barcelona, Spain
Prof. Lina Badimon

Cardiovascular Research Center (CSIC-ICCC), Institut Català de Ciències Cardiovasculars, CIBEROBN-Pathophysiology of Obesity and Nutrition (PI), Hospital de la Santa Creu i Sant Pau, Av. Sant Antoni M. Claret 167, 08025 Barcelona, Spain

J. M. Pou

Servicio de Endocrinología y Nutrición, Hospital de Sant Pau, 08025 Barcelona, Spain

\section{G. Vilahur}

Cardiovascular Research Center (CSIC-ICCC), Institut Català de Ciències Cardiovasculars (ICCC), CIBEROBN-Pathophysiology of Obesity and Nutrition, Hospital Sta. Creu i St. Pau, Av. Sant Antoni Maria Claret, 167, 08025 Barcelona, Spain

\footnotetext{
L. Badimon

Cardiovascular Research Center (CSIC-ICCC), Institut Català de Ciències Cardiovasculars, CIBEROBN-Pathophysiology of Obesity and Nutrition (PI), Hospital de la Santa Creu i Sant Pau, Av. Sant Antoni M. Claret 167, 08025 Barcelona, Spain
} 